\title{
Heterotopic tracheal transplantation with omentum wrapping in the abdominal position preserves functional and structural integrity of a human tracheal allograft
}

\author{
Walter Klepetko, MD \\ Gabriel Mihai Marta, MD \\ Wilfried Wisser, MD ${ }^{\mathrm{a}}$ \\ Enrico Melis, MD \\ Alfred Kocher, MDa \\ Gernot Seebacher, MDa \\ Clemens Aigner, $\mathrm{MD}^{\mathrm{a}}$ \\ Samy Mazhar, MDa
}

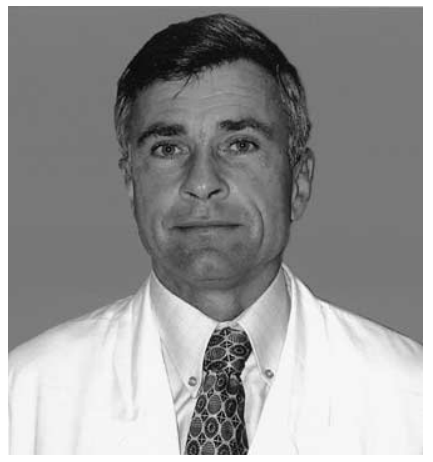

Dr Klepetko

From the Department of Cardiothoracic Surgery, ${ }^{\mathrm{a}}$ University Hospital of Vienna, Vienna, Austria, and Department of Thoracic Surgery, , University of Rome "La Sapienza," Rome, Italy.

Received for publication March 11, 2003; revisions requested March 31, 2003; revisions received May 5, 2003; accepted for publication July 7, 2003.

Address for reprints: Walter Klepetko, MD, University Hospital of Vienna, Department of Cardio-Thoracic Surgery, Waehringer Guertel 18-20, 1090 Vienna, Austria (Email: walter.klepetko@akh-wien.ac.at).

J Thorac Cardiovasc Surg 2004;127:862-7

0022-5223/\$30.00

Copyright (C) 2004 by The American Association for Thoracic Surgery

doi:10.1016/j.jtcvs.2003.07.050
Objectives: Transplantation of a human trachea has been reported only twice in the literature with limited documentation of the functional and structural properties of the allograft.

Patients and Methods: A 57-year-old patient with chronic obstructive pulmonary disease with low segment tracheal stenosis was accepted for lung transplantation and 2-stage tracheal allotransplantation. Standard bilateral sequential lung transplantation was performed with the transfer of the donor trachea into the recipient's abdomen, which was wrapped in the greater omentum and sutured into the abdominal wall, similar to a stoma. The patient received immunosuppression consisting of cyclosporine A, mycophenolate mofetil, and cortisone. Sixty days later, the tracheal allograft presented with macroscopically normal appearance with maintained elasticity and rigidity. The patient underwent a cricotracheal resection 6 months after lung transplantation. However, reconstruction with direct end-to-end anastomosis was achievable. The tracheal allograft, therefore not needed for reconstruction, was harvested and underwent complete investigations.

Results: Cross-section of the graft revealed a mechanically stable and macroscopically intact trachea. Hematoxylin-eosin staining demonstrated vital cartilage covered by respiratory epithelium. Angiography, followed by corrosion studies and electromicroscopy, demonstrated excellent vascularization of the tracheal wall.

Conclusion: The patient is alive 31 months posttransplantation and remains in bronchiolitis obliterans syndrome stage 0. Human trachea wrapped in omentum maintains its functional and structural integrity and may be used for 2-stage allotransplantation.

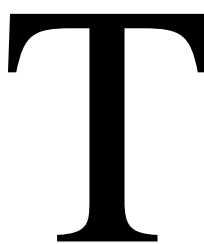

racheal transplantation has been investigated for several decades. Contrary to parenchymal organs, the human trachea is supplied by a network of small vessels, which makes it inaccessible to direct revascularization.

Although a number of different experimental approaches have been tried in the search for solutions for this problem, only 2 reports about transplantation of the trachea in humans have been documented. In 1979, Rose and colleagues ${ }^{1}$ reported the first allogenic tracheal transplantation in a human. The donor trachea was 
implanted heterotopically into the sternocleidomastoid muscle of the recipient and transferred into the orthotopic position after 3 weeks. In 1993, Levashov ${ }^{2}$ described a case of 1-stage allotransplantation of the trachea with omentum wrapping of the graft. Both reports, however, lack a clear documentation of the viability of the allograft and the functional qualities of its different structures.

This case report documents our experience with heterotopic tracheal revascularization by omentum wrapping in the abdominal position. Ultimately, the graft was not needed to accomplish tracheal reconstruction; however, minucious workup demonstrated its structural integrity and suitability for transfer into the orthotopic position.

\section{Clinical Summary}

A 57-year-old man with terminal chronic obstructive pulmonary disease was referred for lung transplantation. He had a history of multiple episodes of respiratory failure, resulting in the repeated need for mechanical ventilation and tracheostomies. Tracheal stenosis developed from these interventions, and multiple treatment attempts with dilatation and laser ablation had been performed.

It was decided to optimize the tracheal situation first and reevaluate the necessity for bilateral lung transplantation only thereafter. At tracheoscopy, the stenotic tracheal segment originated from the level of the cricoids bow and extended downward to a total length of $5 \mathrm{~cm}$. A bougienage of the stenosis was performed, and a Polyflex stent 13 to $50 \mathrm{~mm}$ in length (Ruesch Company, Kernen, Germany) was inserted. This resulted in a widely open and patent tracheal lumen. However, the patient remained severely dyspnoic and hypercapnic. The lung function analysis showed that his $\mathrm{FEV}_{1}$ was $12 \%$ predicted and blood gases were $\mathrm{Po}_{2} 68 \mathrm{~mm} \mathrm{Hg}$ and $\mathrm{PCO}_{2} 56 \mathrm{~mm} \mathrm{Hg}$ under $3 \mathrm{~L}$ of oxygen.

The potential treatment options for the patient were discussed, which included conservative treatment, bilateral lung transplantation only, or a combination of bilateral lung transplantation together with tracheal resection and reconstruction. However, it was expected that resection and direct end-to-end anastomosis of the trachea after lung transplantation might become technically difficult because of the length of the stenotic segment and the limited mobilization of the trachea after the previous transplantation. It was therefore decided to offer the patient a staged procedure, consisting of a bilateral lung transplantation with the transfer of the donor trachea into the recipient's abdomen, wrapped in the greater omentum. This left the option for a later resection of the stenotic tracheal segment followed by either direct reanastomosis or reconstruction with the trachea revascularized and pedicled from the omentum. The choice of this procedure was based on extensive experimental experience published by others.

After a waiting period of 18 months, a suitable donor (identical blood group, total lung capacity predicted within $10 \%$ range of recipient, cytomegalovirus constellation $[-/-]$ identical) became available. The double lung block was harvested together with the trachea up to the larynx. The trachea was then separated from the lungs at the level of the main bronchi, and the bronchi of the lung allografts were closed with staplers for further procurement. Standard bilateral sequential lung transplantation was performed through 2 separate bilateral anterior thoracotomies.

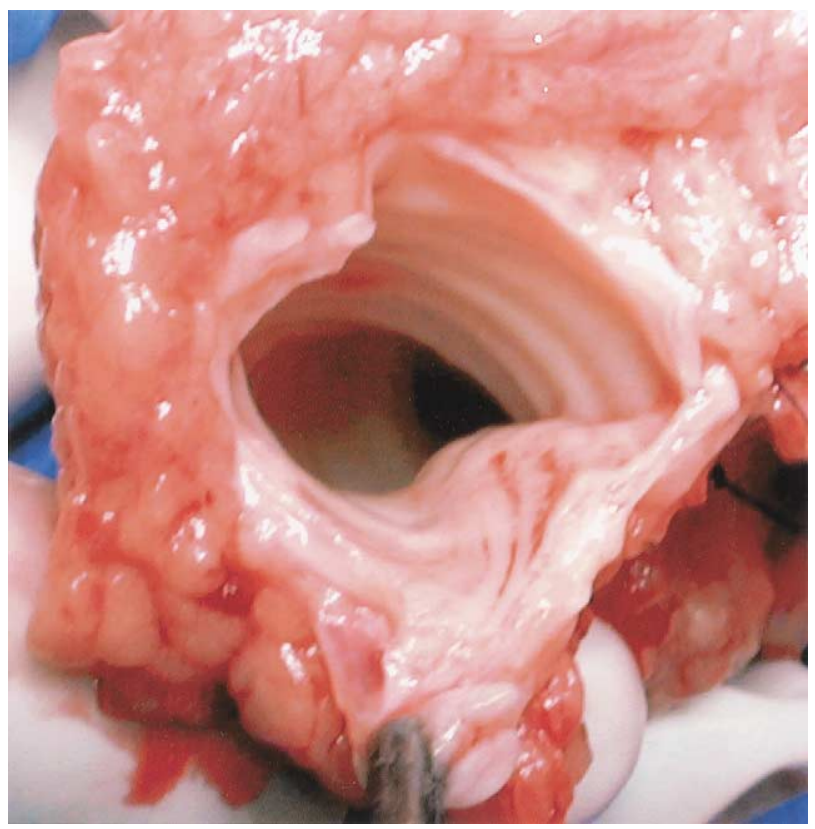

Figure 1. Donor trachea wrapped in the distal part of the great omentum.

Thereafter, the abdomen was opened by a median laparotomy. The donor trachea was closed by staplers at 1 end and wrapped in the distal part of the greater omentum (Figure 1), which was adapted with single 4-0 PDS stitches (Ruesch Company, Kernen, Germany) to have close contact with the trachea. The open end of the trachea was then sutured into the abdominal wall, similar to a stoma, and the laparotomy was finally closed.

The stoma allowed permanent control of vascularization of the graft, clearance of secretions during the postoperative period, and good accessibility for eventual biopsies. In addition, a silicone stent was loosely inserted into the tracheal graft to prevent any potential shrinking.

Tracheostomy of the patient was performed at the end of the operation, and the tracheal stent was removed.

Standard triple immunosuppressive therapy was started with intravenous cyclosporine A, mycophenolate mofetil, and corticosteroids, and was maintained orally thereafter.

The patient was weaned from mechanical ventilation within 5 days and had an uneventful further recovery. After removal of the tracheal tube, a self-expandable silicone stent (Polyflex, Ruesch Company) was inserted into the segment at risk to prevent new stenosis formation.

The tracheal allograft sutured into the abdominal wall was checked daily. This check included removing the stent, cleaning any secretions (which eventually were only minor), and inspecting the mucosal viability. During the first 5 days, there was evidence of progressive ischemia, with the tracheal mucosa becoming increasingly dark. On postoperative day 6 , dark red bleeding from the mucosa started, followed by a stepwise improvement of the macroscopic aspect of the mucosa during the next 2 weeks. Sixty days later the tracheal allograft presented with a macroscopically normal appearance and maintained rigidity and elasticity of the cartilaginous tracheal rings. 


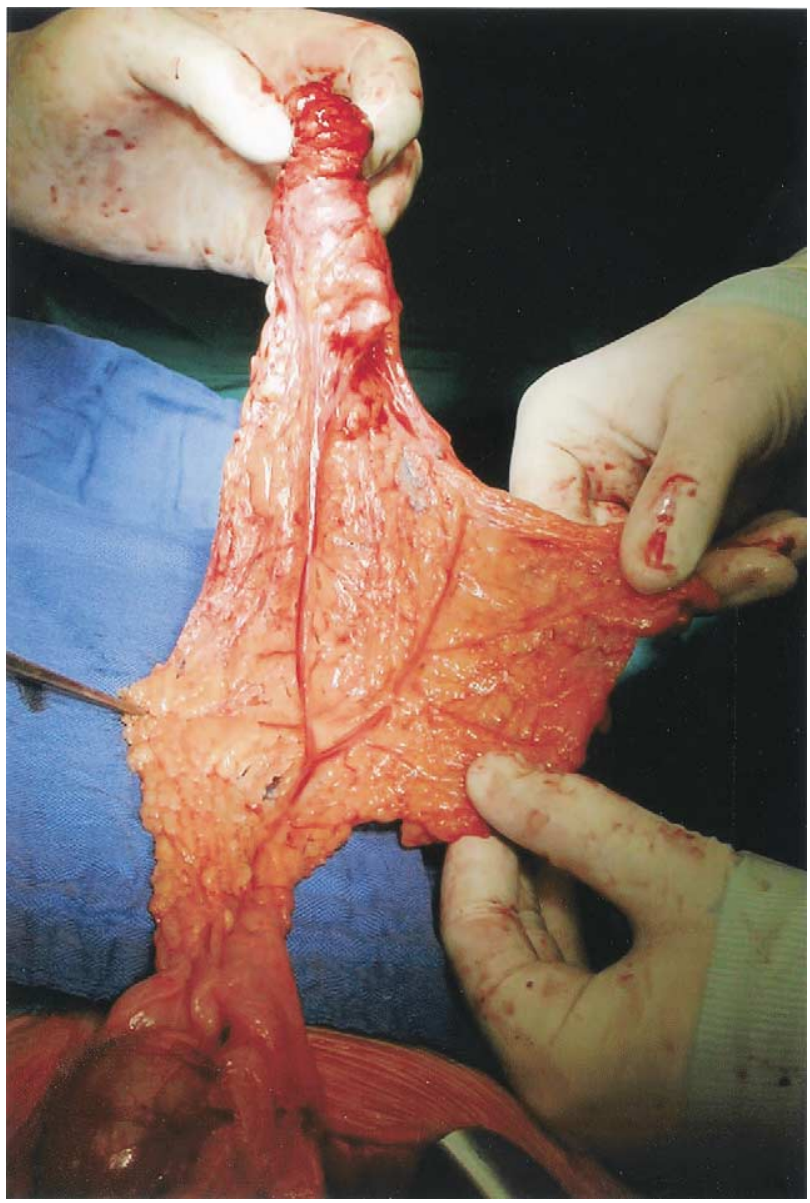

Figure 2. Dissection of the omentum together with the wrapped trachea through an additional laparotomy.

The patient was discharged from the hospital on postoperative day 31. During the further follow-up, recurrent episodes of inflammation around the cervical part of the stent occurred, which made temporary removal of the stent and intravenous antibiotic therapy necessary. Developing restenosis, restenting, and recurring episodes of local infection postponed the definitive attempt to repair the stenotic segment of the trachea, which finally was performed 8 months after the transplantation.

Through a collar incision, a resection of the 5-cm stenotic segment of the trachea together with the anterior cricoid cartilage was performed. The resection was technically difficult because of enormous calcifications resulting from the previous tracheostomies and inflammations. However, after meticulous mobilization of the distal trachea and the larynx, including a suprahyoidal release, a direct thyroid-cricoid-tracheal end-to-end anastomosis was achieved.

The heterotopic trachea in the abdomen was therefore explanted. The stoma was incised circumferentially, and an additional laparotomy was performed, through which the omentum together with the wrapped trachea was dissected (Figure 2). The omentum was pedicled, and it was proved that it had enough length to reach the neck for direct implantation of the trachea. Thereafter,

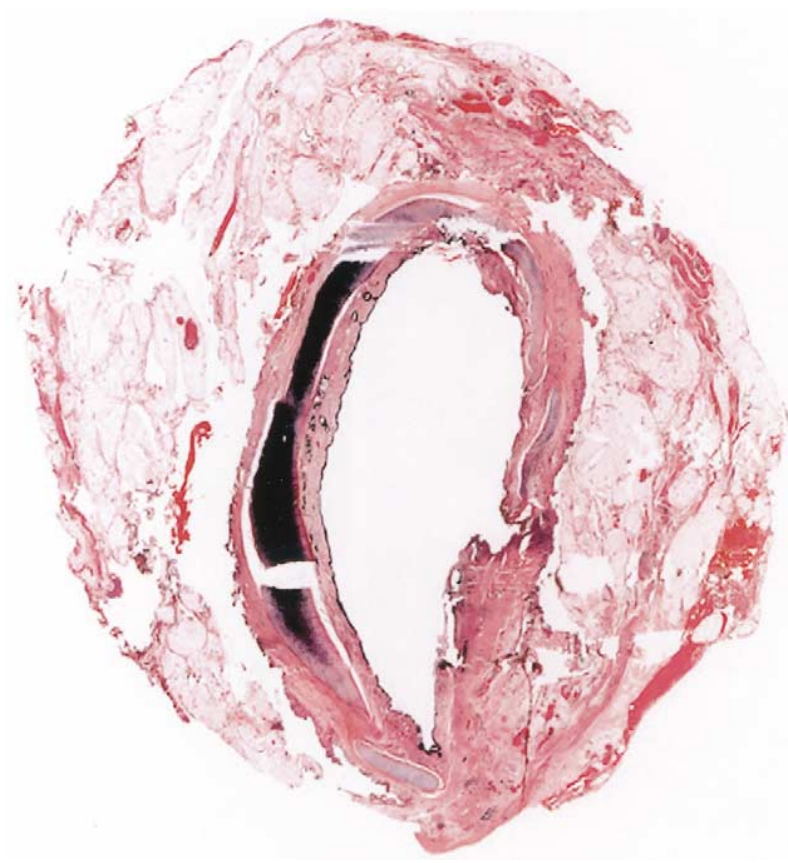

Figure 3. Cross-section revealing mechanically stable and macroscopically intact trachea with a histologically vital cartilage.

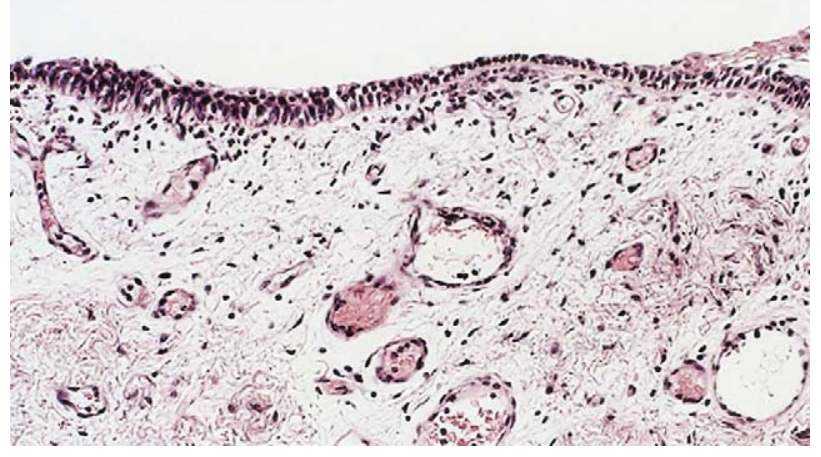

Figure 4. Respiratory epithelium without signs of rejection.

the allograft together with its omental pedicle was harvested for further investigations.

Cross-section of the graft revealed a mechanically stable and macroscopically intact trachea. Hematoxylin-eosin staining revealed vital cartilage covered by respiratory epithelium (Figure 3 ). Angiography, followed by corrosion study and electron microscopy, demonstrated excellent vascularization of the tracheal wall (Figure 4).

The patient had an uneventful postoperative recovery and was discharged from the hospital 6 days after the operation. The laryngotracheal anastomosis healed well, and no restenosis occurred during the further observation period. The patient remains in excellent clinical condition 31 months later and currently is in bronchiolitis obliterans syndrome stage 0 . 


\section{Discussion}

Transplantation of the human trachea remains an unsolved technical problem in thoracic surgery. The main reason is the lack of a well-defined blood supply, which makes direct revascularization a difficult procedure. An overview of the different, most important techniques for this specific pathology in thoracic surgery is listed in Table 1.

Until now, only 2 experimental techniques of direct revascularization have been described in the surgical literature. In 1993, Khalil-Marzouk and Cooper ${ }^{3}$ used a composite thyrotracheal allograft in dogs, with anastomoses of the cranial thyroid arteries to the ipsilateral common carotid arteries, but without venous drainage. The reported results were promising because 5 of 6 vascularized and immunosuppressed grafts showed preservation of tracheal cartilages and surrounding soft tissues. However, the method never gained further acceptance, because the principles of this technique were in discordance with the established surgical experience that a lack of venous drainage ultimately results in infarction of a graft. Macchiarini and colleagues ${ }^{4}$ attempted to overcome this limitation by developing an extensive en bloc technique of thyrotracheal transplantation in pigs. Harvesting consisted of en bloc cervicothoracic exenteration including the aortic arch, supraaortic vessels, anterior vena cava together with the jugular veins, subclavian vessels, thyroid glands, and esophagus. Revascularization was performed by anastomoses of the large arterial and venous vessels. All tracheal grafts remained viable and had preserved or regenerated airway epithelium. However, because of the invasiveness of the procedure, it has not achieved application in humans.

The alternative approach, to revascularize a tracheal graft indirectly by wrapping it with viable tissue and stimulating ingrowth of microvessels, has been intensively investigated in the experimental literature. Preferentially, the greater omentum has been used, although there are some rare reports about the use of other tissues like fascia thoracica ${ }^{5,6}$ or sternocleidomastoideus muscle. ${ }^{1}$ The reason for this is based in the long-established experience with the use of omentum in general thoracic surgery, together with the omentum's unique features of easy surgical handling and excellent potential for induction of neoangiogenesis. ${ }^{7}$ Two different strategies have been applied: orthotopic 1-stage transplantation and heterotopic transplantation into the abdomen followed by transfer into the neck at a second stage.

The orthotopic 1-stage technique was initially described by Baldermann and Weinblatt ${ }^{8}$ in 1987. Nakanishi and colleagues ${ }^{9}$ investigated the maximal length of autografts transplanted with this technique. They were able to demonstrate that grafts longer than $4 \mathrm{~cm}$ frequently showed ischemia in the mid-portion. From this finding, they concluded that the blood supply to the graft derived from both ends and the wrapped omentum concomitantly and that only the synergistic effects of the 2 blood supplies succeeded in preservation of graft viability at both ends but not at the mid-portion of the graft.

On the basis of these findings, the "split transplantation of the trachea" technique, in which the transplanted long segmented trachea was divided at its mid-portion to facilitate blood vessel ingrowth, was reported by Yokomise and colleagues. ${ }^{10}$ No ischemia or stenosis was detected after an observation period of 2 months.
However, in clinical reality, autotransplantation is not feasible, and reduction of alloantigenicity of allotransplants is of importance. Nakanishi and Yasumoto ${ }^{11}$ investigated the impact of immunosuppression in a heterotopic allotransplant model. They proved that application of adequate doses of cyclosporine A after transfer of the donor trachea into the recipient omentum was effective to prevent allograft rejection and guarantee excellent healing.

Another approach for reduction of alloantigenicity of a tracheal transplant was investigated with the use of cryopreserved allografts. ${ }^{12}$ With storage at $-196^{\circ} \mathrm{C}$ for 60 days, cartilage viability was found to be preserved; however, the epithelium was grossly denudated, and no additional immunosuppression was therefore needed. Ultimately, longitudinal shrinking of the grafts was observed after a period of 3 months. These findings were confirmed by Mukaida and colleagues ${ }^{13}$ and Tojo and colleagues ${ }^{14}$ who were able to demonstrate the superiority of cryopreserved allografts to native ones in the absence of immunosuppression. More recently, similar evidence was gained in a nonhuman primate model by Murawaka and colleagues ${ }^{15}$

The potential advantage of a heterotopic implantation of the trachea into the omentum in the abdominal position as a first step was investigated by Li and colleagues. ${ }^{16,17}$ They resected a 6-ring segment of trachea and reconstructed it by end-to-end anastomosis in dogs. The tracheal graft was wrapped in the lower portion of the omentum. Two weeks later, the omental pedicle, together with the tracheal graft, was easily brought to the cervical area and sewed into the former defect. All dogs survived, and the tracheal grafts maintained their lumen with preserved epithelium and cartilage. The authors concluded that the prior implantation of tracheal grafts into the omentum results in better preservation of its structure and that reconstruction of a tracheal defect with a tracheal graft implanted first into the abdomen is feasible.

In contrast with the wide experimental experience, few reports about tracheal replacement with allografts in humans exist. Most of the reports describe the use of cryopreserved tracheal homografts, which were used to reconstruct long segmental stenotic defects. This procedure, initially developed by Herberhold and colleagues, ${ }^{18}$ however, implies that the homografts are split longitudinally on their posterior portion and that a rim of the native trachea is preserved and sewed into the defect from where recipient tracheal epithelium grows into the graft. Temporary stenting, until complete reepithelialization has occurred, is part of the treatment plan, whereas no immunosuppression is necessary because of reduced immunogenicity. The total North American and worldwide pediatric experience with this technique was recently reviewed by Jacobs and colleagues, ${ }^{19}$ with 26 of 31 children surviving and only 1 reported treatment failure.

Except for this elegant approach, which still relies on the presence of functional tracheal epithelium in the posterior part of the recipient's trachea, only 2 cases of allotransplantation of a whole trachea have been reported. In 1979, Rose and colleagues ${ }^{1}$ published a report on the heterotopic implantation of a donor trachea that was wrapped in the sternocleidomastoid muscle and transferred into the orthotopic position 3 weeks later. Despite the fact that the authors did not add any immunosuppressive therapy, a satisfying initial result after 9 weeks was reported, with no 
TABLE 1. Experimental approaches in humans and animals

\begin{tabular}{|c|c|c|}
\hline Experiences in animals & Advantages & Limitations \\
\hline \multicolumn{3}{|l|}{ Direct vascular anastomosis } \\
\hline Composite thyrotracheal transplant ${ }^{3}$ & $\begin{array}{l}\text { Preservation of arterial } \\
\text { blood supply }\end{array}$ & No venous drainage \\
\hline Heterotopic thyrotracheal allograft model ${ }^{4}$ & $\begin{array}{l}\text { Preservation of arterial and } \\
\text { venous blood supply }\end{array}$ & Invasiveness of procedure \\
\hline \multicolumn{3}{|l|}{ Indirect revascularization } \\
\hline \multicolumn{3}{|l|}{ Omentum wrapping } \\
\hline Orthotopic 1 stage 8,11 & & $\begin{array}{l}\text { Limitation in length of } \\
\text { graft (ischemia) }\end{array}$ \\
\hline $\begin{array}{l}\text { Heterotopic } 2 \text { stage }{ }^{17} \\
\text { (Olech et al. Ann Thorac Surg 1991) } \\
\text { (Borro et al. EJCTS 1992) }\end{array}$ & $\begin{array}{l}\text { Preservation of } \\
\text { respiratory } \\
\text { epithelium } \\
\text { Morphologic excellent } \\
\text { results }\end{array}$ & \\
\hline \multicolumn{3}{|l|}{ Fascial wrapping (Delaere et al. JTCVS 1995) } \\
\hline Split transplantation of the trachea ${ }^{10}$ & & Clinical relevance \\
\hline \multicolumn{3}{|l|}{ Sternohyoid muscle wrapping (de Mello- Filho } \\
\hline \multicolumn{3}{|l|}{ Experiences in humans } \\
\hline $\begin{array}{l}\text { Heterotopic 2-stage sternocleidomastoid wrapping, no } \\
\text { immunosuppression }{ }^{1}\end{array}$ & $\begin{array}{l}\text { No rejection, ischemia, } \\
\text { infection }\end{array}$ & $\begin{array}{l}\text { Lack of histologic } \\
\text { documentation }\end{array}$ \\
\hline Orthotopic 1- stage omentum wrapping with IS² & & Progressive stenosis \\
\hline \multicolumn{3}{|l|}{$\begin{array}{l}\text { Experiences with cryopreserved allografts (no } \\
\text { immunosuppressants) } \\
\text { Nonhumans }{ }^{13-15}\end{array}$} \\
\hline (Moryama et al. Ann Thorac Surg 2000) & $\begin{array}{l}\text { Reduction of acute } \\
\text { rejection } \\
\text { No stenosis } \\
\text { Preservation of structural } \\
\text { integrity } \\
\text { Preservation of pars } \\
\text { cartilaginosa }\end{array}$ & $\begin{array}{l}\text { Shrinking of the grafts; } \\
\text { chronic rejection }\end{array}$ \\
\hline \multirow[t]{2}{*}{$\begin{array}{l}\text { Humans }^{19} \\
\text { (Herberhold et al. EJCTS 1996) }\end{array}$} & & $\begin{array}{l}\text { Stenosis and sepsis; } \\
\text { transplantation only of } \\
\text { pars cartilaginosa }\end{array}$ \\
\hline & $\begin{array}{l}\text { Encouraging short- to } \\
\text { medium-term results; } \\
\text { expectation of good } \\
\text { long-term results }\end{array}$ & \\
\hline
\end{tabular}

evidence of rejection, ischemia, or infection. However, no further information on the long-term results exists.

The second case of human tracheal transplantation was reported by Levashov and colleagues ${ }^{2}$ in 1993. A 24-year-old female patient with idiopathic fibrosing mediastinitis, affecting the thoracic segment of the trachea, received an allograft replacement of the distal trachea together with omentum wrapping and cyclosporine A-based immunosuppression. Early rejection after 10 days was diagnosed and treated with augmented immunosuppression, resulting in good functional outcome after 2 months. However, beginning from the fourth postoperative month, progressive stenosis occurred, and the patient ultimately needed permanent stenting of the trachea. It remains unclear whether this outcome was the result of late shrinking of the graft because of ischemia or of underlying disease progression.

In view of this combined experimental and clinical experience, it was decided to offer our patient the previously described pro- cedure. A number of arguments were in favor of this decision. Most important, the final need for a tracheal transplantation was unclear at the time of evaluation. Long segmental tracheal stenting after bilateral lung transplantation was expected to create a significant risk for recurrent infections. In fact, repeated localized infectious episodes had to be overcome before a definitive repair was attempted. In addition, the length of the stenotic tracheal segment would have already been borderline accessible to resection and direct end-to-end anastomosis in a healthy patient. However, after bilateral lung transplantation, a significant fixation of the distal trachea was expected, possibly preventing the necessary mobilization for tension-free end-to-end anastomosis. With the graft readily available in the abdomen, surgical solutions for all situations were prepared.

A simultaneous replacement of both lungs and the trachea was never considered because of all these reasons and the invasiveness of the procedure. 
To offer the patient the highest possible safety, we created an abdominal stoma with 1 end of the graft, which allowed permanent check of the tracheal vascularization and the possibility of inserting a silicon stent. How important this stent really was for prevention of allograft shrinking remains unclear. In fact, a minimal longitudinal shortening of the graft was observed at the time of harvesting, although this was not precisely measured.

The final analysis of the explanted allograft evidenced the viability and structural integrity of the heterotopic transplanted trachea. In addition, the feasibility for a transfer of the pedicled graft into the cervical region was proven. However, from this experience, the minimal level of immunosuppression needed in humans for prevention of tracheal graft rejection remains unclear, because this patient received routine triple drug therapy at a level comparable to that received by other lung transplant patients.

\section{Conclusion}

This is the first human case of heterotopic tracheal allotransplantation and omentum wrapping in the abdomen. All necessary prerequisites for a final transfer of the graft into the orthotopic position were sufficiently achieved. This experience could serve as a model for a planned tracheal transplantation in treating long extending, nonmalignant tracheal problems.

\section{References}

1. Rose KG, Sesterhenn K, Wustrow F. Tracheal allotransplantation in man. Lancet. 1979;1:433.

2. Levashov YN, Yablonsky PK, Cherny SM, Orlov SV, Shafirovsky BB, Kuznetzov IM. One stage allotransplantation of thoracic segment of the trachea in a patient with idiopathic fibrosing mediastinitis and marked tracheal stenosis. Eur J Cardiothorac Surg. 1993;7:383-6.

3. Khalil-Marzouk JF, Cooper JD. Allograft replacement of the trachea. Experimental synchronous revascularization of composite thyrotracheal transplant. J Thorac Cardiovasc Surg. 1993;105:242-6.

4. Macchiarini P, Lenot B, de Montpreville V, Dulment E, Mazmanian GM, Fattal M, et al. Heterotopic pig model for direct revascularization and venous drainage of tracheal allografts. J Thorac Cardiovasc Surg. 1994;108:1066-75.

5. Delaere PR, Liu ZY, Feenstra L. Tracheal autograft revascularization and transplantation. Arch Otolaryngol Head Neck Surg. 1994;120: 1130-6.

6. Delaere PR, Liu ZY, Hermans R, Sciot R, Feenstra L. Experimental tracheal allograft revascularization and transplantation. J Thorac Cardiovasc Surg. 1995;110:728-37.

7. Morgan E, Lima O, Goldberg M, Ferdman A, Luk SK, Cooper JD. Successful revascularization of totally ischemic bronchial autografts with omental pedicle flaps in dogs. J Thorac Cardiovasc Surg. 1982; 84:204-10.

8. Balderman SC, Weinblatt G. Tracheal autograft revascularization. J Thorac Cardiovasc Surg. 1987;94:434-41.

9. Nakanishi R, Shirakusa T, Mitsudomi T. Maximum length of tracheal autografts in dogs. J Thorac Cardiovasc Surg. 1993;106:1081-7.

10. Yokomise H, Inui K, Wada H, Ueda M, Hitomi S, Itoh H. Split transplantation of the trachea: a new operative procedure for extended tracheal resection. J Thorac Cardiovasc Surg. 1996;112:314-8.

11. Nakanishi R, Yasumoto K. Minimal dose of cyclosporin A for tracheal allografts. Ann Thorac Surg. 1995;60:635-9.

12. Moriyama H, Sasajima T, Hirata S, Yamazaki K, Yatsuyanagi E, Kubo Y. Revascularization of canine cryopreserved tracheal allografts. Ann Thorac Surg. 2000;69:1701-6.

13. Mukaida T, Shimizu N, Aoe M, Andou A, Date H, Okabe K, et al. Experimental study of tracheal allotransplantation with cryopreserved grafts. J Thorac Cardiovasc Surg. 1998;116:262-6.

14. Tojo T, Niwaya K, Sawabata N, Kushibe K, Nezu K, Taniguchi S, et al. Tracheal replacement with cryopreserved tracheal allograft: experiment in dogs. Ann Thorac Surg. 1998;66:209-13.

15. Murawaka T, Nakajima J, Noburu M, Murakami A, Tamamoto S. Successful allotransplantation of cryopreserved tracheal grafts with preservation of the pars membranacea in nonhuman primates. J Thorac Cardiovasc Surg. 2002;123:153-60.

16. Li J, Xu P, Chen H, Yang Z, Zhang Q. Improvement of tracheal autograft survival with transplantation into the greater omentum. Ann Thorac Surg. 1995;60:1592-6.

17. Li J, Xu P, Chen H. Successful tracheal autotransplantation with two-stage approach using the greater omentum. Ann Thorac Surg. 1997;64:199-202.

18. Herberhold C, Stein M, Falkenhausen M. [Long-term results of homograft reconstruction of the trachea in childhood.] Laryngorhinootologie. 1999;78:692-6.

19. Jacobs JP, Quintessenza JA, Andrews T, Burke RP, Spektor Z, Delius RE, et al. Tracheal allograft reconstruction: the total North American and worldwide pediatric experiences. Ann Thorac Surg. 1999;68: $1043-52$. 